\title{
Do financial incentives increase treatment adherence in people with severe mental illness? A systematic review
}

\author{
ALEXANDRA BURTON, STAMATINA MAROUGKA, STEFAN PRIEBE \\ Queen Mary University of London, Barts and the London School of Medicine and Dentistry, Unit for Social and Community \\ Psychiatry, London (United Kingdom)
}

SUMMARY. Aim - To identify whether financial or material incentives improve treatment adherence in people with severe mental illness. Method - A systematic review of studies published between 1950 and 2008 was conducted. EMBASE, MEDLINE, EBM, AMED and PsycINFO were searched. Studies were included if a financial or material incentive was offered and if the sample had a severe mental illness. Results - Fourteen articles were identified; three studies on adherence to psychiatric treatment and one on physical exercise. Ten articles used incentives for adherence to substance misuse treatment programmes. In all studies, financial incentives were associated with an increase in adherence; however the effect was not always maintained once the incentive was withdrawn. Conclusion - While existing research suggests that financial incentives may improve treatment adherence in severely mentally ill populations, very few studies focus on psychiatric treatment. Further research may address the long term effectiveness of incentives on adherence in this population.

Declaration of Interest: The authors on this paper were supported by funds from the Wellcome Trust. All authors worked on a Wellcome Trust funded qualitative focus group study exploring stakeholder views on offering patients financial incentives to adhere to antipsychotic medication. Priebe is also lead applicant on a National Institute of Health Research (England) (NIHR) awarded grant to conduct a clinical trial on the use of financial incentives to achieve maintenance antipsychotic medication adherence.

KEY WORDS: patient non-adherence, health behaviour, review, systematic, incentives.

Received 15.10.2009 - Final version received 01.12.2009 - Accepted 12.12.2009.

\section{INTRODUC'TION}

Non-adherence to medication and treatment programmes by people with mental health problems is particularly common (Fenton et al., 1997; Lacro et al., 2002; Nosé et al., 2003) and is associated with a range of negative outcomes. It may result in poorer outcomes for the individual, for example, increased rates of relapse and rehospitalisation, as well as increasing the burden on health services. The healthcare costs of patients with schizophrenia who do not adhere to medication are reportedly higher than the costs for those that do adhere (Gilmer et $a l ., 2004)$. It has been suggested that non-compliance

Address for correspondence: Dr. A. Burton, Unit for Social and Community Psychiatry, Newham Centre for Mental Health, Cherry Tree Way, Glen Road, London, E13 8SP (United Kingdom).

Fax: +44-20-7540 2976

E-mail: a.burton@qmul.ac.uk with treatment is a feature of at least a quarter of suicides and homicides by people with mental health problems (Appleby, 2000).

Several strategies such as compliance therapy, psychotherapy, family education, telephone prompting and psycho-education have been tested to improve adherence to maintenance antipsychotic medication. Studies show that they have at best, a limited effect (McDonald et al., 2002; O'Donnell et al., 2003). Adherence therapy, which aims to improve compliance with medication, is specifically not recommended for people with schizophrenia (National Institute of Clinical Excellence, 2009). Against this background, the question arises as to what initiatives may be effective in improving adherence to treatment.

Research conducted in the United States has shown that financial incentives can considerably improve adherence to treatment in a range of conditions. A systematic review found that 10 out of 11 randomised controlled studies using financial incentives described positive results for anti-tuberculosis drugs, dental care, weight 
reduction, cocaine dependence, and anti-hypertensive treatment (Giuffrida \& Togerson, 1997). None of these studies were conducted with patients with psychiatric disorders; however a clinical trial is currently underway in the UK which seeks to examine the effectiveness of financial incentives on adherence to anti-psychotic maintenance medication (Priebe et al., 2009).

Patient experiences of the use of leverage such as money or housing to improve adherence to treatment in psychiatric patients in the community have been explored (Monahan et al., 2005). Half of the individuals reported that a form of leverage had been used to encourage adherence to treatment, with the provision of accommodation being the most commonly used incentive.

There has been some ethical debate over the use of financial incentives to achieve adherence in people with mental health problems. While financial incentives could be perceived as coercive (Shaw, 2007), others claim that they should be interpreted as a reward and could be viewed as less coercive than existing forms of leverage, such as enforced hospital admission (Burns, 2007). While this controversial debate is likely to continue for conceptual, ethical and practical reasons, the debate should be informed by evidence on the use of financial incentives for treatment adherence in severely mentally ill populations.

\section{Aims of the study}

To systematically review the existing published literature on whether financial or material incentives improve adherence to treatment in people with severe mental illness.

\section{MATERIAL AND METHODS}

An initial broad search for articles was undertaken to identify all studies where financial and material incentives have been used to achieve adherence to treatment in people with severe mental illness. Articles where financial or material incentives were offered to severely mentally ill patients to improve adherence to substance misuse treatment programmes were included in the review.

Relevant studies were located through a search of the following databases up to October 2008: EBM (1991+), PsychINFO (1950+), EMBASE (1980+), Medline (1950+) and AMED (1985+). The following search terms were combined simultaneously to identify relevant studies: 'incentive', 'money', 'payment' 'contingency management', 'voucher', 'financial' with the terms 'medication', 'therapy', 'appointment' 'compliance' 'adherence' and 'mental health', 'mental illness', or 'psychiatr' to identify studies specific to severely mentally ill populations.

The reference lists of relevant articles identified through this process were hand searched to identify any further research articles. All four authors of the mental health treatment specific articles were contacted to identify whether any other relevant studies existed. Three responded and identified no further studies beyond those included in this review. Ten key psychiatry journals including Acta Psychiatrica Scandinavica, American Journal of Psychiatry, Archives of General Psychiatry, British Journal of Psychiatry, Journal of Clinical Psychiatry, Epidemiologia E Psichiatria Sociale, Psychopharmacology, Journal of Social Psychiatry and Psychiatric Epidemiology, Schizophrenia Bulletin and Psychiatric Services were manually searched for relevant articles from the previous five years (January 2004January 2009), but yielded no further results.

Papers were selected for inclusion in the review using the following criteria: the incentive had to be a direct offer of payment to an individual in the form of a monetary, voucher based or material goods value before they received treatment. The impact the incentive had on adherence had to be reported and participants had to be diagnosed with a severe mental illness. Data were extracted from each study by two research workers and compared to ensure reliability of the extraction tool. Where discrepancies appeared, these were discussed until a consensus was reached.

Due to the expectation that limited research would be found in this area, the inclusion criteria for study methodologies was not restricted to randomised controlled trials. A Meta Analysis was not conducted on the data, as it was anticipated that studies would be too heterogeneous with regards to the interventions, treatment settings, participant diagnoses, and outcomes measured. A descriptive synthesis of the results is therefore presented.

\section{RESULTS}

From the databases searched, 90 articles were retrieved for further screening (See Figure 1). Fifty-four articles were excluded as they referred specifically to people with substance misuse disorders with no other diagnosed mental health problem. A further 28 articles were excluded as the incentive was not of a financial or material value. Eight studies selected for inclusion were identified directly from the database search. In one of these studies (Messina et al., 2003), participants either had a substance 
misuse problem or a diagnosis of antisocial personality disorder (ASPD) and substance misuse problem. Only the outcomes from the ASPD group are taken into account for the purposes of this review. The remaining six articles were identified through a manual search of the bibliographies from articles with a related title.

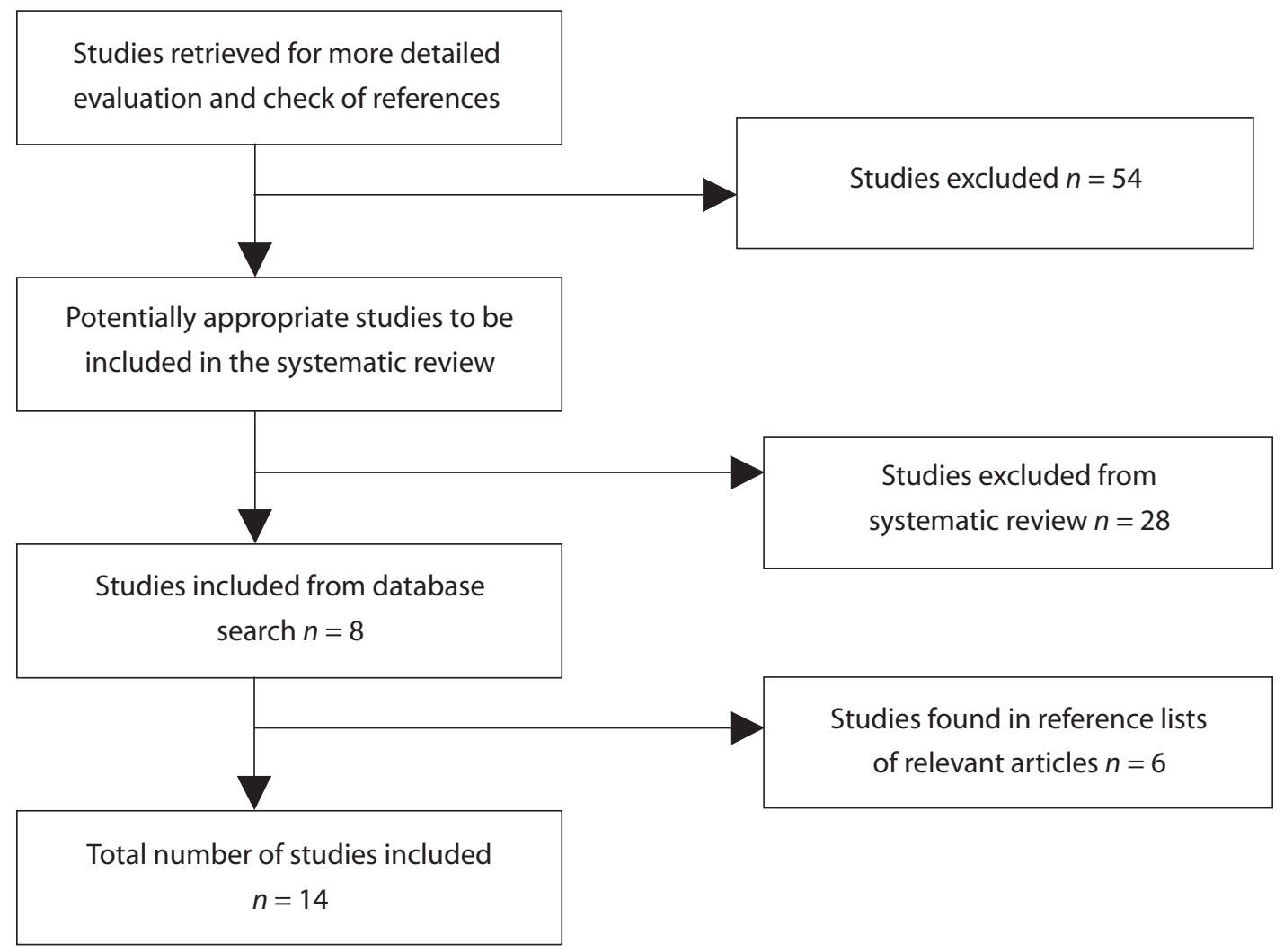

Figure 1 - QUOROM diagram.

From the 14 articles that met the inclusion criteria, three sought to encourage adherence to mental health specific treatments (Claassen et al., 2007; Post et al., 2006; Olson \& Greenberg, 1979). This included adherence to depot medication for people with schizophrenia (Claassen et al., 2007), attendance at therapy sessions for people with depression (Post et al., 2006), and attendance and active participation in group meetings for people with schizophrenia and other disorders (Olson \& Greenberg, 1979). One study aimed to encourage the uptake of physical exercise in people with schizophrenia (Thyer et al., 1984). The remaining ten studies offered financial or material incentives to people with severe mental illness to encourage abstinence from substances and/or adherence to substance misuse treatment programmes. This included two studies on abstinence from smoking in people with schizophrenia (Carey \& Carey, 1990; Tidey et al., 2002). See Tables I and II for study details.

\section{Study Participants}

Study participants were either outpatients, living in independent accommodation or hostels in the community, living in the community and accessing assertive outreach services, or psychiatric inpatients. In seven of the studies, participants were diagnosed with schizophrenia or other psychotic disorders. One study included people with depression (Post et al., 2006) while another included people with ASPD (Messina et al., 2003). In the remaining five studies, participants had a range of mental disorders. 


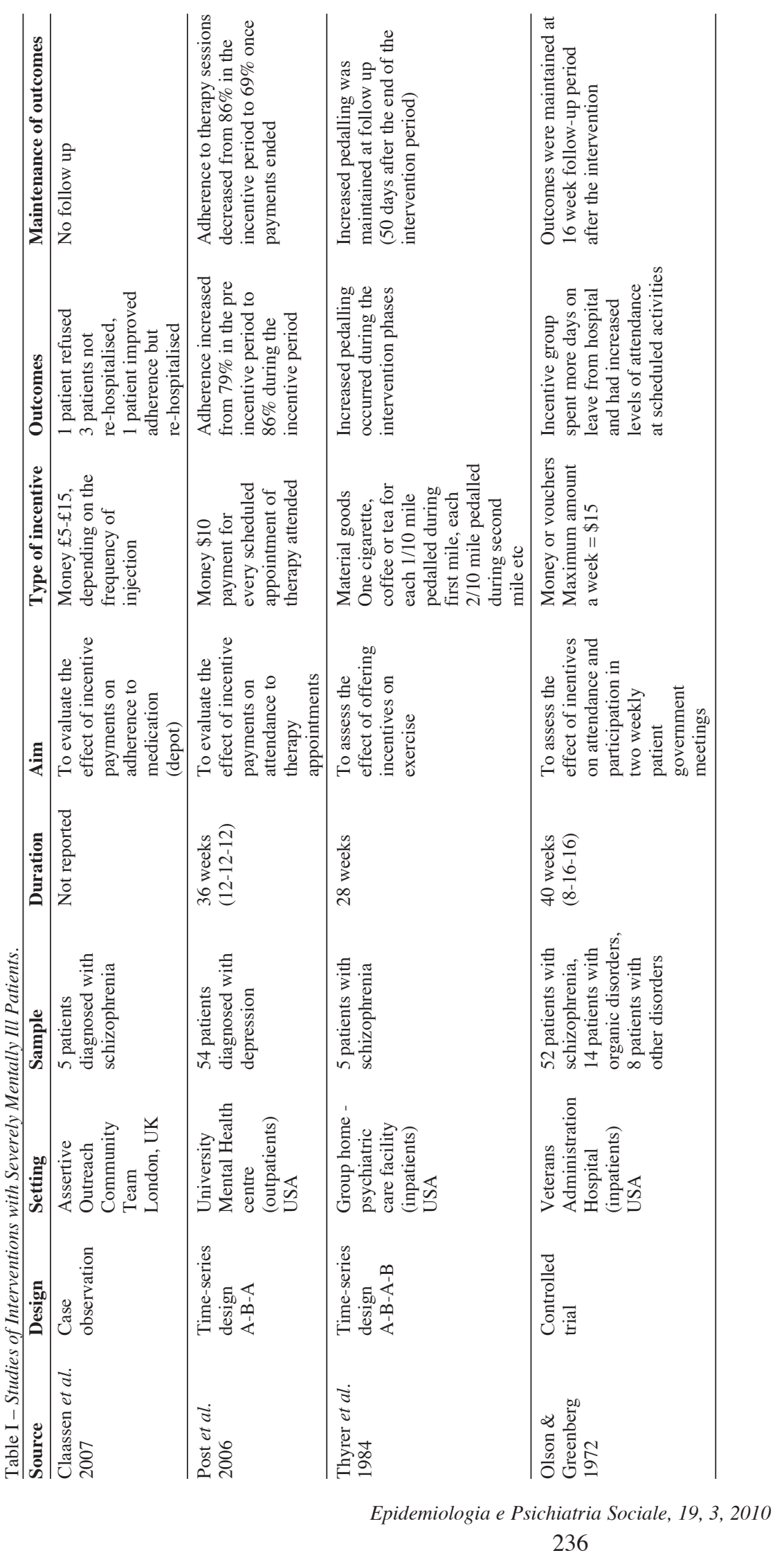


Do financial incentives increase treatment adherence in people with severe mental illness? A systematic review
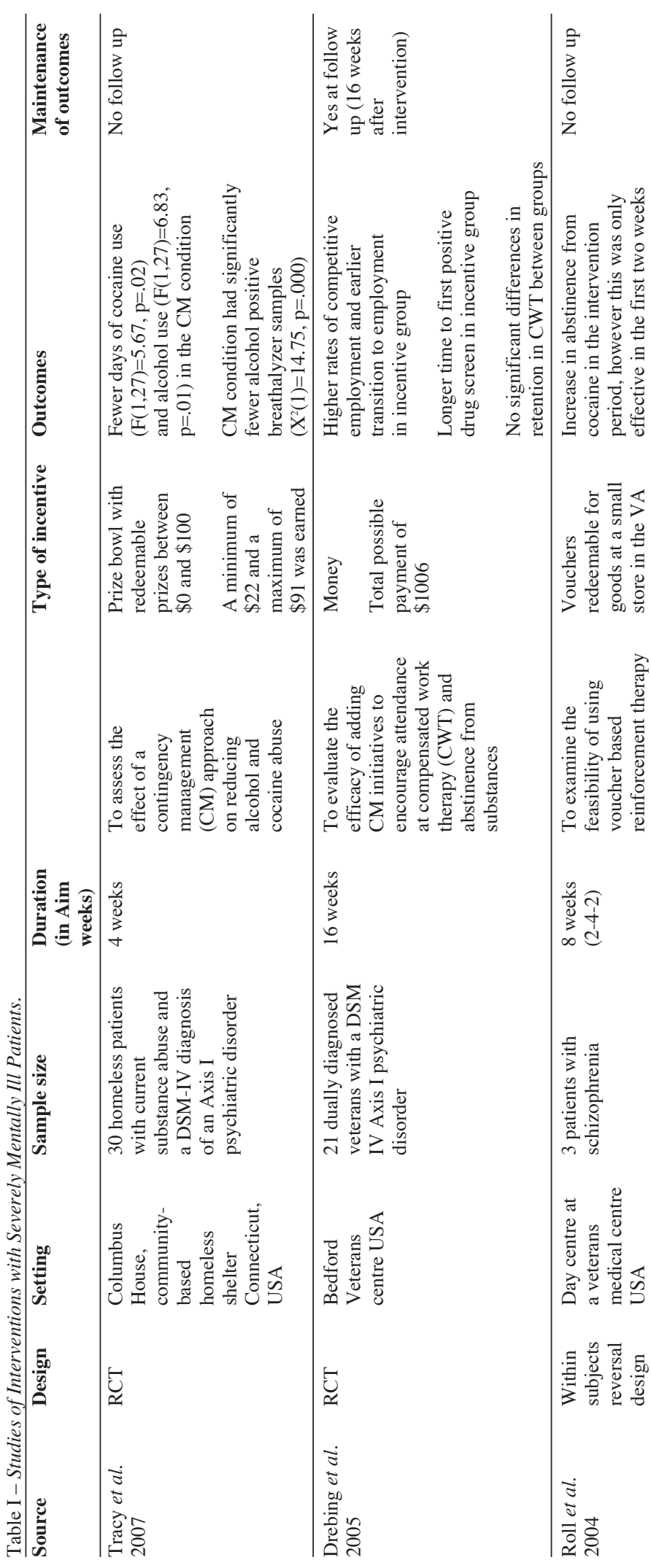

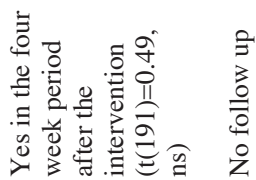

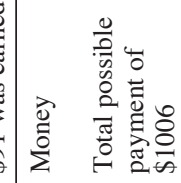

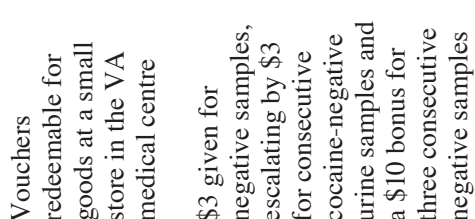

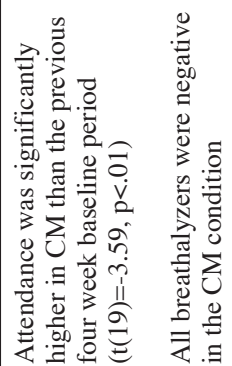
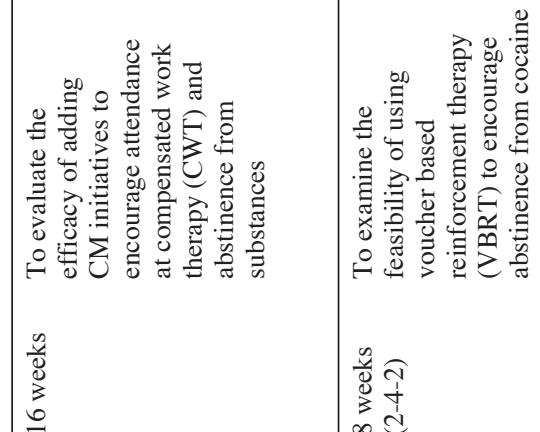

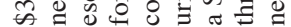

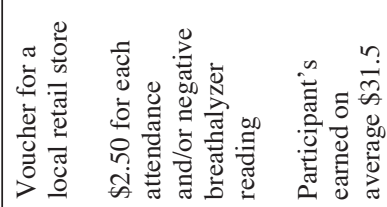
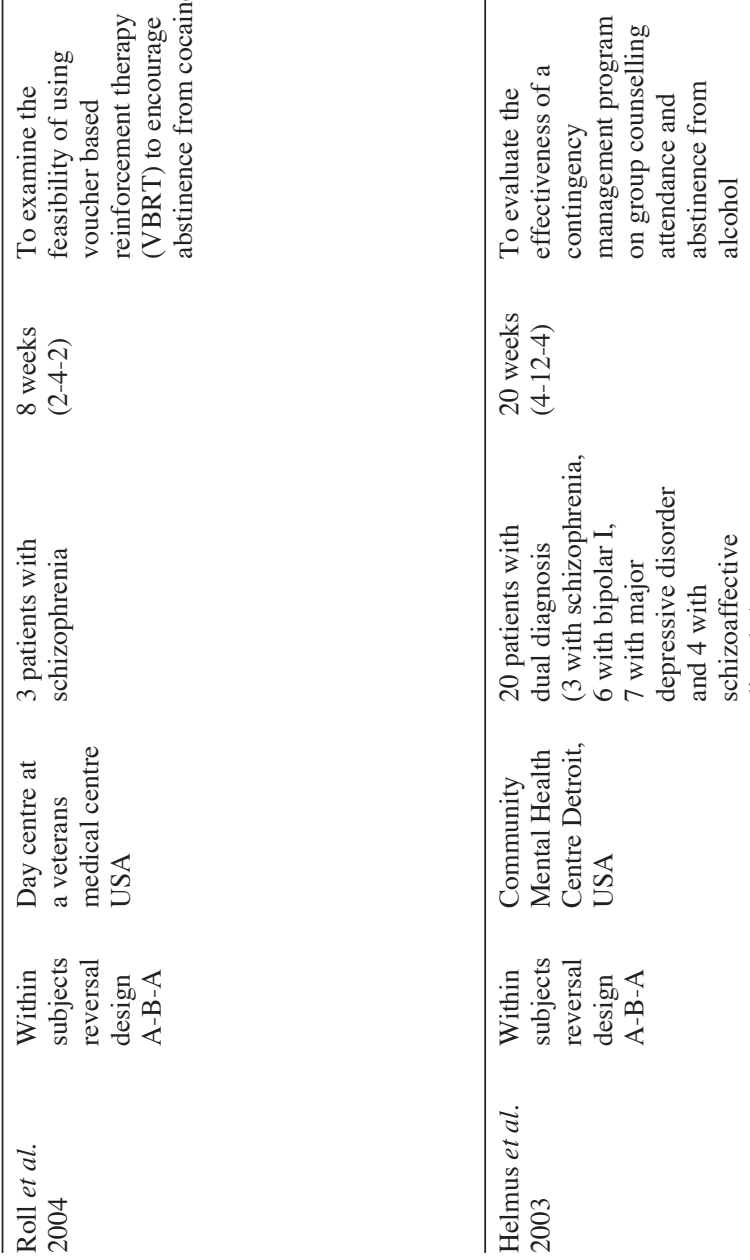

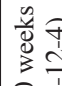

斿

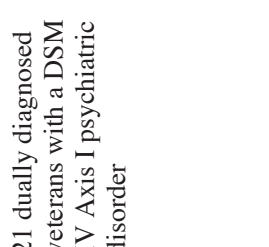

$\overrightarrow{\mathrm{N}}$
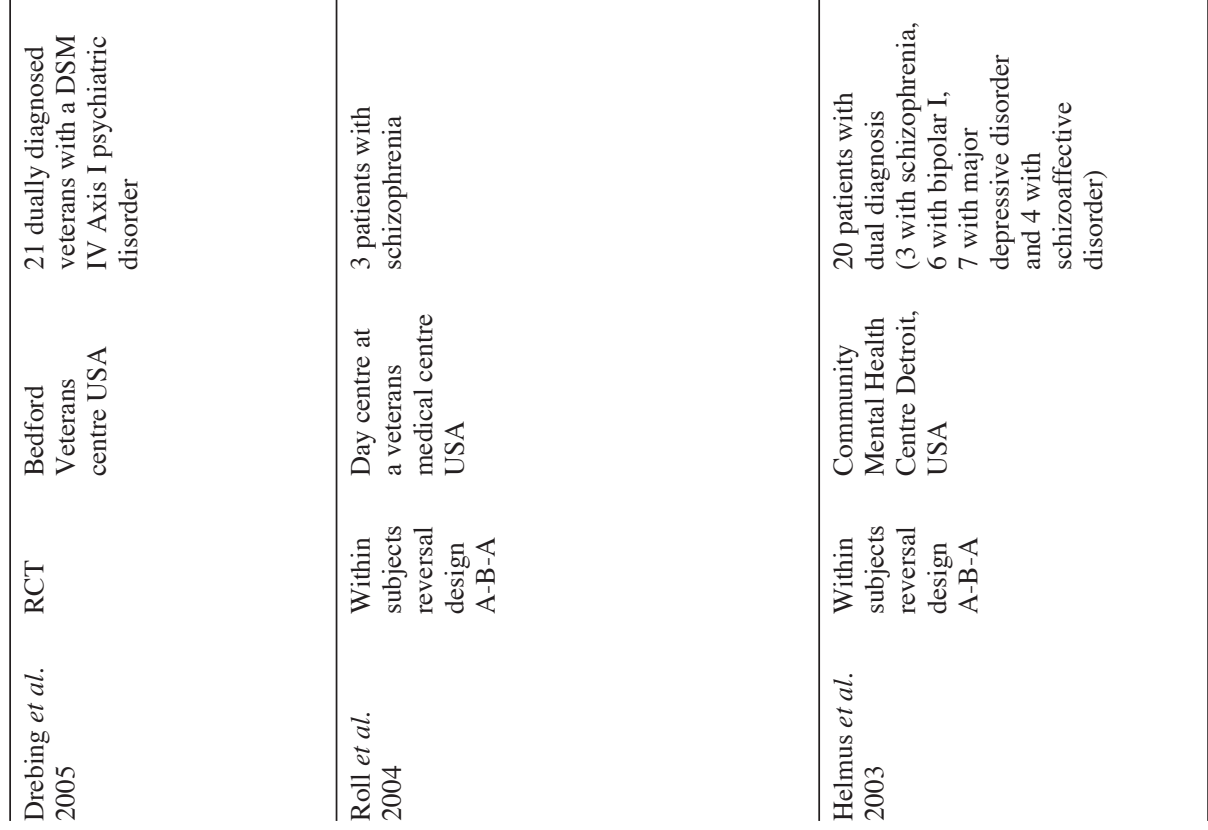

Epidemiologia e Psichiatria Sociale, 19, 3, 2010 

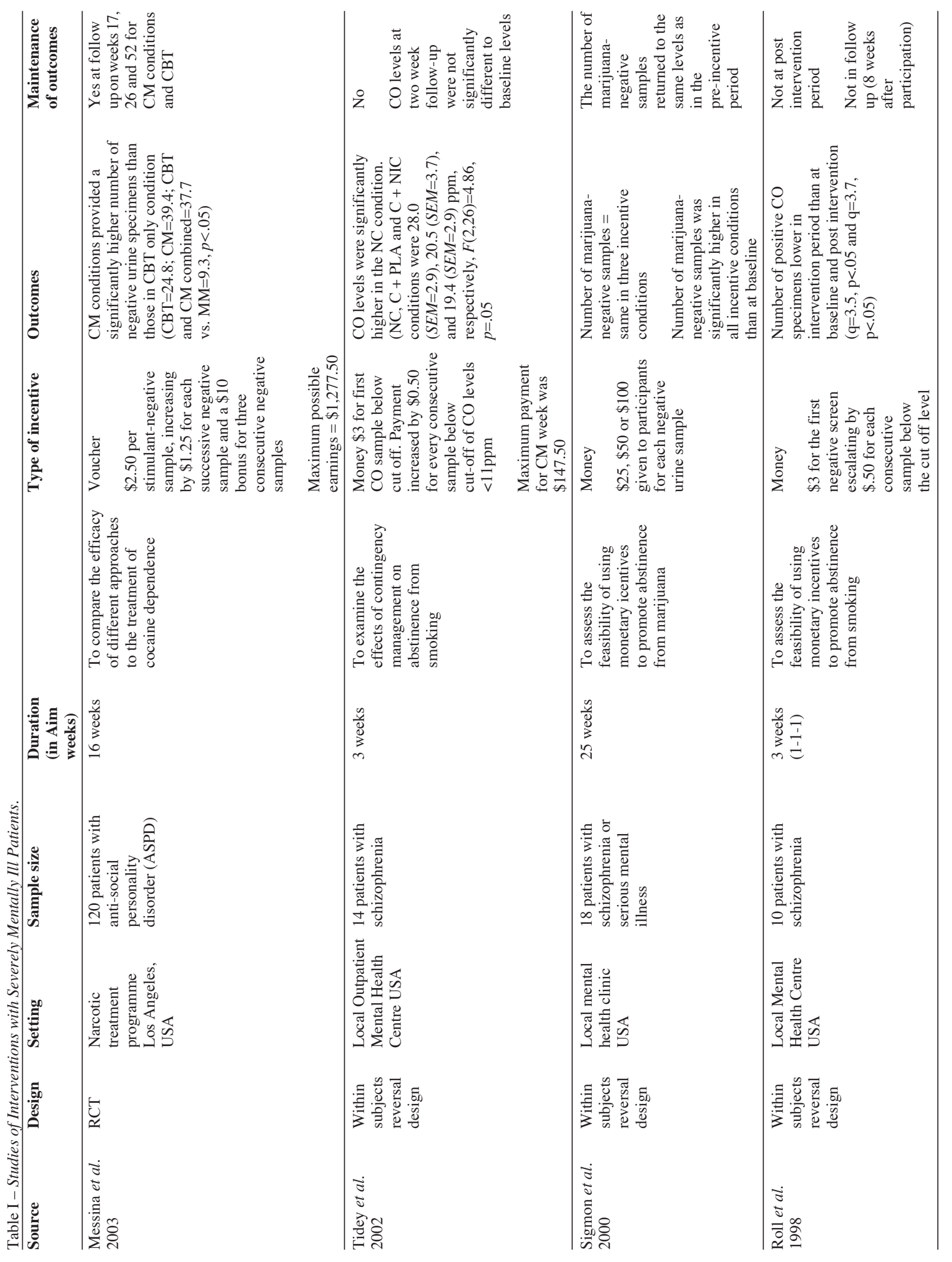

Epidemiologia e Psichiatria Sociale, 19, 3, 2010 


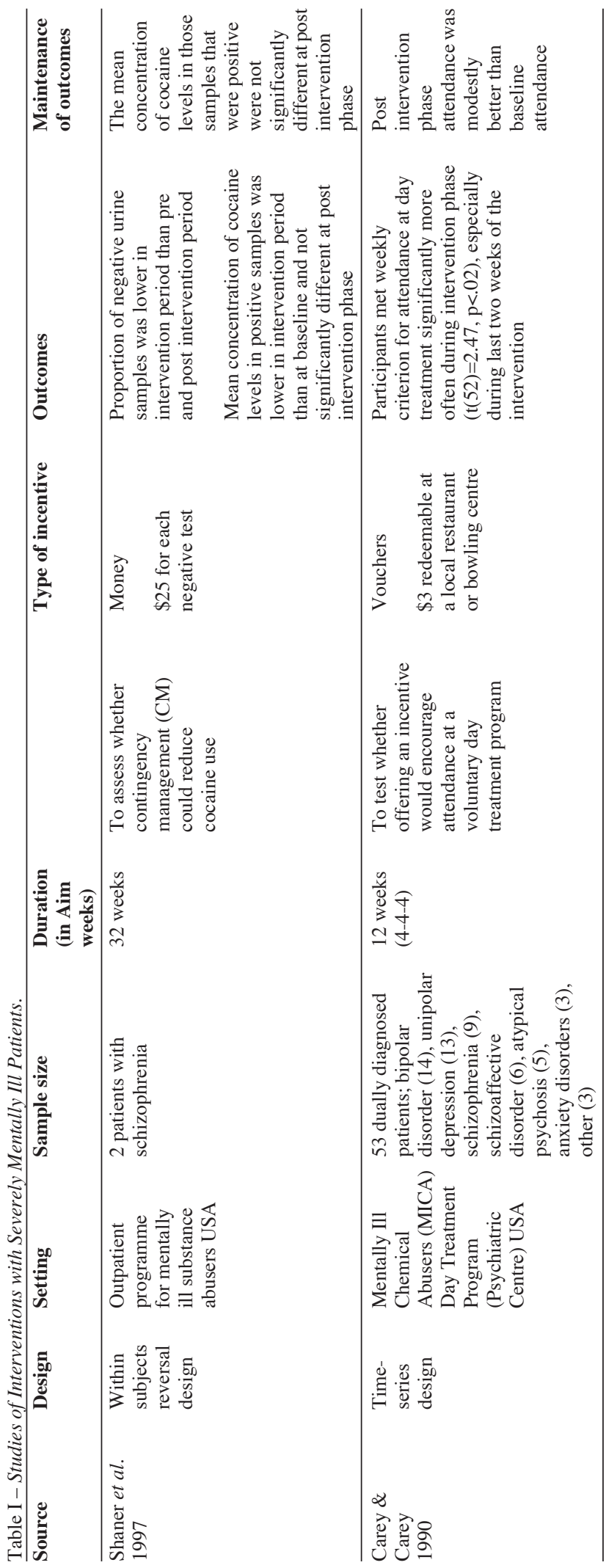

\section{Study Design}

Nine studies utilised a time series study design with no control group. There was one case observation study (Claassen et al., 2007) one controlled trial for active involvement in inpatient group meetings (Olson \& Greenberg 1979) and three randomised controlled trials; two examining the effect of offering incentives to promote abstinence from substances (Messina $e t$ al., 2003, Tracy et al., 2007), and one for a combination of attendance at compensated work therapy and abstinence from substances (Drebbing $e t$ al., 2005).

In three out of the ten studies aiming to reduce substance and alcohol misuse, the incentive was given for participation in therapy as well as for the provision of a drug negative sample. All studies were conducted in the United States with the exception of one UK based study (Claassen et al., 2007), and were undertaken between 1972 and 2007. The intervention periods lasted between one week and 40 weeks with one study not specifying an end point (Claassen et al., 2007), The sample sizes varied from between two and 120 patients, with nine of the studies containing a sample size of less than 30 participants.

\section{Incentives used}

The type of incentive used was either in the form of a direct payment of money or vouchers and in one study, money or vouchers alongside three bus passes a week (Olson \& Greenberg, 1979). The maximum possible incentive that could be earned in each study ranged from $\$ 12$ to $\$ 1,277.50$. Where vouchers were offered, these could be exchanged for goods either in the shops of each centre, or on activities that were estimated to be valued by the participants, such as fast food, bowling and days out. Two studies used material goods as the incentive. Participants were given an extra allowance of tea, coffee or cigarettes or could win prizes from a lucky dip. An extra payment was given independent of the intervention in seven of the studies, usually after participants completed baseline assessments.

In the three studies focusing on psychiatric treatment, patients were offered $£ 5-£ 15$ per depot

Epidemiologia e Psichiatria Sociale, 19, 3, 2010 
injection received (Claassen et al., 2007), \$10 per therapy appointment attended, usually one a week for a 12week period (Post et al., 2006), and a maximum of $\$ 15$ dollars a week for a 16 week period (Olson \& Greenberg, 1979) dependent on attendance and participation in patient group meetings.

\section{Outcomes}

All of the studies found that the use of financial incentives can improve adherence to mental health treatment or abstinence from substances. The results show that during the intervention phase, when the incentive was offered, adherence to treatment and abstinence from substances improved when compared with baseline assessments, with eleven of the fourteen studies reporting this improvement as significant. Six out of the eleven studies that included a post intervention assessment demonstrated an improvement in adherence from the first baseline assessment; where measurements were recorded before the incentive was introduced, and the post intervention phase; where the incentive had been taken away and adherence was measured again. This improvement was only reported as significant in one of the studies. Messina et al., 2003 demonstrated that abstinence from cocaine remained significantly higher at 52 week follow up (between $71 \%$ and $80 \%$ of cocaine samples provided were negative in the incentive conditions compared with $20 \%$ in the control group).

Claassen et al. (2007) reported that four out of five patients who were offered a financial incentive to adhere to depot medication accepted; three had improved adherence to medication after money was offered and were not re-hospitalised during the incentive phase. The forth participant had improved adherence, however was re-hospitalised. Post et al. (2006) found that attendance at therapy sessions significantly increased from $79 \%$ in the pre incentive period, to $86 \%$ during the intervention phase, however this significantly decreased to $69 \%$ once the incentive payments were discontinued. Thyer et al. (1984) found that participants increased the amount of physical exercise they did during the intervention phase and that this was maintained 50 days after the incentive was withdrawn (no statistical analyses reported) while Olsen \& Greenburg (1972) reported that the incentive group spent more days on leave from hospital than the control groups at 16 week follow up and had a significantly increased level of attendance at scheduled groups during the incentive period (no follow up).

\section{DISCUSSION}

The literature in this review suggests that offering financial or material incentives to people with a severe mental illness can improve adherence to treatment during the period of time in which the incentive is offered. Where follow up is documented however, five out of the eleven studies that carried out a post intervention assessment suggest that adherence deteriorates back to baseline levels or worse once the incentive is taken away. The remaining six studies maintained improved adherence, however only one study demonstrated significantly improved adherence levels when compared o baseline assessments (Messina et al., 2003).

The strengths of this review are the systematic nature in which articles were extracted. We utilised a range of search methods and search terms including database and manual journal searches, as well as contacting experts to verify that no other published research on this topic existed.

The weaknesses of this review are the lack of homogeneity between studies, making it difficult to combine outcome measures and thus form a coherent conclusion applicable to a clearly defined population. Nevertheless we wanted to be as broad as possible in our search for articles specific to the severely mentally ill population due to a presumed lack of research in the field.

Financial incentives have been found to be successful in modifying a range of health behaviours (Jochelson, 2007), therefore the potential they may have in influencing adherence to treatment among people with mental health problems should not be underestimated. Research specific to mental health settings is however limited to a small number of studies, with most of the research aimed at improving adherence to substance misuse treatment programmes and the provision of drug negative samples.

There are also methodological problems with the majority of studies included in this review. There are very few randomised controlled trials on financial incentives in this population, and 10 of the studies included in this review lacked a control group. Small sample sizes may impact on the generalisabilty of results to a wider population. Many studies do not include a follow up period beyond a post intervention assessment immediately after the incentive has been withdrawn, meaning that only short term conclusions regarding effectiveness can be reached. Three of the studies do not report statistical analyses on the data; therefore the significance of these results cannot be reported. 
The finding in five of the studies, that adherence levels decline once the incentive is taken away, raises a practical issue as to the length of time incentive schemes should be implemented in order to achieve long-term treatment compliance. This is not addressed by the existing research. A consideration of why patients may discontinue treatment once incentives are withdrawn is also absent from the research. Nose 2008 suggests that future research on medication adherence should take in to consideration the impact of side effects from both the patient and clinician perspective. What the existing research does suggest however is that even an offer of a small financial incentive has the potential to increase adherence to treatment during that time.

All but one of the studies were conducted in United States healthcare settings. The transferability of results to European models of healthcare needs to be treated with caution. For example, although the assertive outreach model appears to be effective in the United States, it was found to be no more effective than the care provided by community mental health teams in the UK (Burns et al., 2007; Killaspy et al., 2006). In the same way, financial incentives may not have the same impact when utilised outside of the United States insurancebased healthcare system.

Concerns have been raised around the acceptability of offering financial incentives to achieve mental health treatment adherence. Post et al. (2006) claim that it may be difficult to implement an incentive scheme at policy level because of differing opinions on whether patient payments are acceptable. The authors also reported that $52 \%$ of participants would attend therapy sessions for money even if they found the session unhelpful, which brings into question the impact that incentives may have on patient outcomes beyond simply turning up to treatment sessions. Olsen \& Greenberg (1972) found that although attendance at group sessions increased with the introduction of incentives, patients in the incentive group demanded that the incentive program stop because they felt that their human rights were being deprived.

The results from this review suggest that financial incentives could be used as an effective tool for initiating engagement in mental health treatment and abstinence from substances. Further robust research is required to determine the long term effectiveness of financial incentives on improving adherence to treatment, as well as the impact incentives may have on patient outcomes, patient attitudes to treatment and the potential effect incentives may have on the therapeutic relationship between patient and clinician.

\section{REFERENCES}

Appleby L. (2000). Safer services: conclusions from the report of the National Confidential Inquiry. Advances in Psychiatric Treatment 6, $5-15$.

Burns T. (2007). Is it acceptable for people to be paid to adhere to medication? Yes. British Medical Journal 335, 232-233.

Burns T., Catty J., Dash M., Roberts C., Lockwood A. \& Marshall M. (2007). Use of intensive case management to reduce time in hospital in people with severe mental illness: systematic review and metaregression. British Medical Journal 335, 336-336.

Carey K.B. \& Carey M.P. (1990). Enhancing the treatment attendance of mentally ill chemical abusers. Journal of Behavior Therapy and Experimental Psychiatry 21, 205-209.

Claassen D., Fakhoury W.K., Ford R. \& Priebe S. (2007). Money for medication: Financial incentives to improve medication adherence in assertive outreach. Psychiatric Bulletin 31, 4-7.

Drebbing C.E., Van Ormer A., Krebs C., Rosenheck R., Rounsaville B., Herz L. \& Penk W. (2005). The impact of enhanced incentives on vocational rehabilitation outcomes for dually diagnosed veterans. Journal of Applied Behavior Analysis 38, 359-372.

Fenton S.W., Blyler C.R. \& Heinssen R.K. (1997). Determinants of medication compliance in schizophrenia: empirical and clinical findings. Schizophrenia Bulletin 23, 637-651.

Gilmer T.P., Dolder C.R., Lacro J.P., Folsom D.P., Lindamer L., Garcia P. \& Jeste D.V. (2004). Adherence to treatment with antipsychotic medication and health care costs among Medicaid beneficiaries with schizophrenia. American Journal of Psychiatry $161,692-699$.

Giuffrida A. \& Togerson D.J. (1997). Should we pay the patient? Review of financial incentives to enhance patient compliance. British Medical Journal 315, 703-707.

Helmus T.C., Schoener E.P. \& Roll J.M. (2003). Reinforcement of counselling attendance and alcohol abstinence in a community based dual-diagnosis treatment program: a feasibility study. Psychology of Addictive Behaviors 17, 249-251.

Jochelson K. (2007). Paying the Patient. Improving Health Using Financial Incentives. Kings Fund.

Killaspy H., Bebbington P., Blizard R., Johnson S., Nolan F., Pilling S. \& King M. (2006). The REACT study: randomised evaluation of assertive community treatment in north London. British Medical Journal 332, 815-20.

Lacro J.P., Dunn L.B. \& Dolder C.R. (2002). Prevalence of and risk factors for medication nonadherence in patients with schizophrenia: a comprehensive review of recent literature. Journal of Clinical Psychiatry 63, 892-909.

Mcdonald H.P., Garg A.X. \& Haynes R.B. (2002). Interventions to enhance patient adherence to medication prescriptions. Scientific review. Journal of the American Medical Association 288, 28682879.

Messina N., Farabee D. \& Reason R. (2003). Treatment responsivity of cocaine-dependent patients with antisocial personality disorder to cognitive-behavioural and contingency management interventions. Journal of Consulting and Clinical Psychology 71, 320-329.

Monahan J., Redlich A.D., Swanson J., Clark Robbins P., Appelbaum P.S., Petrila J., Steadman H.J., Swartz M., Angell B. \& McNiel D.E. (2005). Use of leverage to improve adherence to psychiatric treatment in the community. Psychiatric Services 56, 37-44.

National Institute for Clinical Excellence. (2009). Schizophrenia. Core Interventions in the Treatment and Management of Schizophrenia in Primary and Secondary Care (Update). NICE: London.

Nosè M. (2008). Tolerability of antipsychotic drugs: does patient perspective deserve consideration? Epidemiologia e Psichiatria Sociale $17,182-185$.

Nosé M., Barbui C., Gray R. \& Tansella M. (2003). Clinical interventions for treatment non-adherence in psychosis: meta-analysis. British Journal of Psychiatry 183, 197-206. 
O’Donnell C., Donohoe G., Sharkey L., Owens N., Migone M., Harries R., Kinsella A., Larkin C. \& O'Callaghan E. (2003). Compliance therapy: A randomised controlled trial in schizophrenia. British Medical Journal 327(7419), 834.

Olson P.R.\& Greenberg D.J. (1979). Effects of contingency-contracting and decision-making groups with chronic mental patients. Journal of Consulting and Clinical Psychology 38, 376-383.

Post E.P., Cruz M. \& Harman J. (2006). Incentive payments for attendance at appointments for depression among low-income African Americans. Psychiatric Services 57, 414-416.

Priebe S., Burton A., Ashby D., Ashcroft R., Burns T., David A., Eldridge S., Firn M., Knapp M. \& McCabe R (2009). Financial incentives to improve adherence to anti-psychotic maintenance medication in non-adherent patients - a cluster randomised controlled trial (FIAT), BMC Psychiatry [online]. Retrieved April 12, 2009, from http://www.biomedcentral.com/1471$244 \mathrm{X} / 9 / 61$

Roll J.M., Higgins S.T., Steingard S. \& Mcginley M. (1998) Use of monetary reinforcement to reduce cigarette smoking of persons with schizophrenia: a feasibility study. Experimental and Clinical Psychopharmacology 6, 157-161.

Roll J.M., Chermack S.T. \& Chudzynski J.E. (2004). Investigating the use of contingency management in the treatment of cocaine abuse among individuals with schizophrenia: a feasibility study.
Psychiatry Research 125, 61-64.

Shaner A., Roberts L.J., Eckman T.A., Tucker D.E., Tsuang J.W., Wilkins J.N. \& Mintz J. (1997). Monetary reinforcement of abstinence from cocaine among mentally ill patients with cocaine dependence. Psychiatric Services 48, 807-810.

Shaw J. (2007). Is it acceptable for people to be paid to adhere to medication? No. British Medical Journal 335, 232-233.

Sigmon S.C., Steingard S., Badger G.J., Anthony S.L. \& Higgins S.T. (2000). Contingent reinforcement of marijuana abstinence among individuals with severe mental illness: a feasibility study. Experimental and Clinical Psychopharmacology 8, 509517.

Thyer B.A., Irvine S. \& Santa C.A. (1984). Contingency management of exercise by chronic schizophrenics. Perceptual Motor Skills 58, $419-425$.

Tidey J.W., O'Neill S.C. \& Higgins S.T. (2002). Contingent monetary reinforcement of smoking reductions, with and without transdermal nicotine, in outpatients with schizophrenia. Experimental and Clinical Psychopharmacology 10, 241-247

Tracy K., Babuscio T., Mich C., Kiluk B., Carroll K.M., Petry N.M. \& Rounsaville B.J. (2007). Contingency management to reduce substance use in individuals who are homeless with co-occurring psychiatric disorders. American Journal of Drug and Alcohol Abuse 33, 253-258. 